For reprint orders, please contact: reprints@futuremedicine.com

\title{
Essential tremor is not a neurodegenerative disease
}

Ali H Rajput ${ }^{* 1}$, Charles H Adler ${ }^{2}$, Holly A Shill ${ }^{3}$ \& Alex Rajput ${ }^{1}$
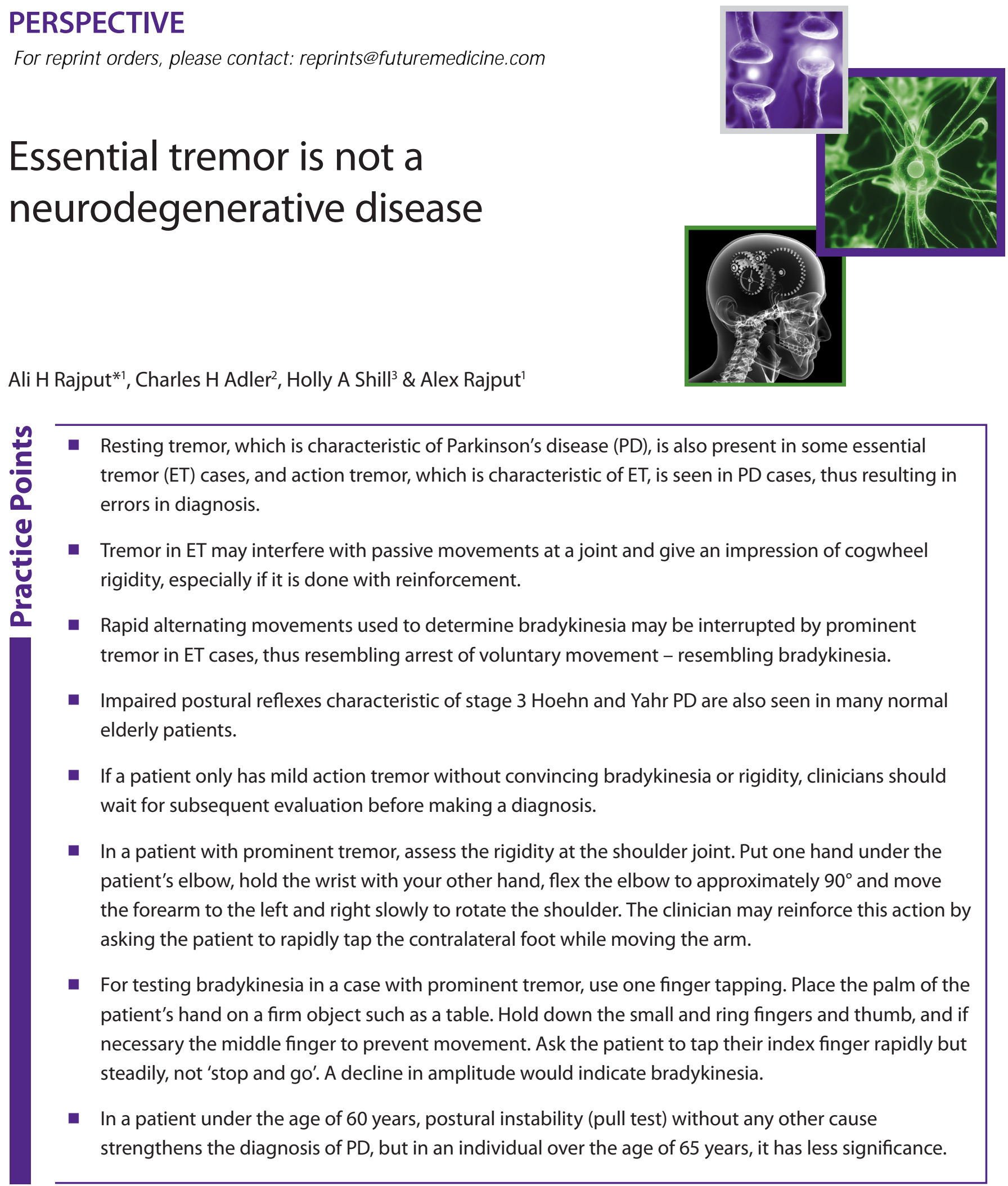

'Movement Disorders Program, Neurology Division, University of Saskatchewan/Saskatoon Health Region, 103 Hospital Drive, Saskatoon, SK, S7N OW8, Canada

2Parkinson's Disease \& Movement Disorders Center, Mayo Clinic Scottsdale, 13400 East Shea Boulevard, Scottsdale, AZ 85259, USA ${ }^{3}$ Banner Sun Health Research Institute, 10515 W. Santa Fe Drive, Sun City, AZ 85351, USA

*Author for correspondence: Tel.: +1 306966 8009; Fax: +1 306 966 8030; ali.rajput@saskatoonhealthregion.ca

Future fss 
- Improvement of tremor with alcohol, $\beta$-blockers or the other drugs used for ET is not sufficiently specific to make a diagnosis of ET.

- Levodopa is prescribed to some ET patients owing to error in diagnosis. If a patient on levodopa has no convincing evidence of bradykinesia, rigidity, wearing-off or dyskinesia, the clinician should gradually withdraw the drug and re-evaluate the patient after 2-3 months. Some patients may resist going off medication because of perceived benefit. If the clinician cannot convince patients to stop taking levodopa, they should assure the patient that it is not toxic to the brain.

- There is no consensus on the duration of ET symptoms before a second diagnosis of PD can be made. The longer the duration of well-documented ET before parkinsonian manifestations, the more accurate the second diagnosis would be. If there is an ET patient who has been well documented without bradykinesia or rigidity for 10 years, and now manifests all three motor features - resting tremor, bradykinesia and rigidity (preferably asymmetrical) - the second diagnosis of parkinsonism is justified.

SUMMARY The pathophysiology of essential tremor (ET) remains unknown. Standard neuropathological studies have reported no consistent changes but a detailed study found neurodegeneration in all ET cases - 24\% demonstrated lower brainstem Lewy body (LB) inclusions and $76 \%$ experienced a loss of cerebellar Purkinje cells (PCs) and its sequelae. We review the evidence on neurodegeneration in ET. The prevalence of LB inclusions in ET brains is similar to that in the asymptomatic general population. These incidental LB disease cases have evidence for reduced striatal tyrosine hydroxylase levels, as found in Parkinson's disease, but there is no evidence for reduced tyrosine hydroxylase levels in ET patients. Reduced mean PC counts in ET cases compared with the controls reported by some studies could not be replicated by others. Most ET cases have the same number of PCs as controls of a comparable age. Neither the lower brainstem LB inclusions nor the cerebellar PC loss represent the neurodegenerative basis of ET. Further studies are needed to determine the pathophysiology of ET.

Tremor is the most common movement disorder [1] and essential tremor (ET) is the most prevalent tremor disorder in the general population $[2,3]$. ET is characterized by action tremor which has two main features: postural tremor (PT) and kinetic tremor (KT). When the KT accentuates on reaching the intended target it is known as terminal or intention tremor. Approximately $20-30 \%$ of ET cases may also manifest resting tremor, usually late in the course of the illness [4-6]. The PT and the KT may be of equal severity in ET or one may be more prominent than the other [7]. Because the life expectancy in ET patients is normal [8], it was frequently classified as 'benign' ET [9]. However, many of these patients develop significant functional disability; therefore, the term 'benign' is not justified $[10,11]$. The other common causes of tremor in neurology practice are: enhanced physiological tremor, medication-induced tremor, Parkinson's disease (PD) and tremor associated with cerebellar disorders.
Diseases characterized by progressive neuronal degeneration in the brain and/or spinal cord are classified as neurodegenerative diseases. The neuronal loss is not consequent to trauma, infection, ischemia, toxins or any other known insult. A well-known example of a neurodegenerative disease is PD [12]. The characteristics of neurodegenerative diseases include: an insidious-onset disease, a threshold of neuronal loss reached before the onset of clinical manifestations $[12,13]$, the loss of disease-specific neurons being considerably accelerated compared with normal aging [12] and the number is progressively smaller compared with age-matched controls and the neuronal loss correlates with the severity of clinical manifestations [14]. Therefore, at the end of life, which is typically several years after the clinical onset, the neuronal loss is so marked that a pathological diagnosis is reasonably easy to make $[12,14,15]$; the neuronal loss at the specific anatomical sites is distinguishable from other disorders that are not known for the loss of those neurons [16]. 
Most reports using standard neuropathological methods found no consistent abnormality in the brain $[4,6]$. However, some recent, more detailed studies, have suggested neurodegeneration in ET [17-20] in the form of either lower brainstem Lewy body (LB) inclusions or cerebellar Purkinje cell (PC) loss and its sequelae [17,18].

The focus of this article will be a review of the literature regarding whether there are pathological findings indicating that ET is a neurodegenerative disorder $[17,18]$. The other related issues will be discussed only briefly where pertinent.

\section{ET \& brain LB inclusions}

LB inclusions and marked substantia nigra neuronal loss are characteristic neuropathological findings in PD [15,21-23]. Tremor is a well known clinical feature in both PD and ET. Striatal dopamine transporters (DATs) [24-26] reveal a dopamine deficit in PD but not in ET. The DAT imaging patterns, however, may not distinguish between ET and several other forms of tremor or between PD and other variants of Parkinson syndrome [24]. Facilities for DAT studies are not available at every center, therefore, the diagnoses of probable ET and PD are often based on clinical features alone $[5,6,15,27]$. ET and PD are both common disorders in the general population. In a community-based study of individuals aged 65 years or older, $14 \%$ had ET and 3\% had PD [28]. Thus, coexistence of the two disorders may occur by chance alone. The complicated cases that manifest features of both ET and the PD are more likely to seek medical care in specialized movement disorder clinics.

The risk of ET patients developing PD has been studied by different groups [29-31]. One large clinical study from two movement disorder centers found that the risk of parkinsonian features in the ET cases was similar to the general population [29]. Another study observed a 24-times higher risk of developing PD in the ET cases [30]. These variations may be due to case selection bias and the use of clinical criteria to make the additional diagnosis of PD. A clinicopathological study reported no increase in the risk of PD in the ET cases [31]. They recommended that the second diagnosis of PD in a pre-existing case of ET should only be made when there is unequivocal evidence of resting tremor, bradykinesia and rigidity, and preferably these findings should be asymmetrical [31].

To our knowledge, there is no literature indicating that the combination of marked substantia nigra neuronal loss and LB inclusions, the characteristics of $\mathrm{PD}$, are also present in ET. One group reported an autopsy study of ten ET cases and found LB inclusions to be present in six $(60 \%)$ cases, however, two patients had pathological findings consistent with PD [19]. A second report from this group found LB inclusions in $24 \%$ of ET cases [18]. A subsequent larger study by Louis et al., which included 40 ET participants, found LB inclusions in only $15 \%$ of cases [20]. In all these studies the LB inclusions in the ET cases were localized to the lower brainstem, primarily the locus coeruleus (LC) [18].

$\mathrm{LB}$ inclusions have also been reported in the brains of individuals who had no known neurological disease at the time of autopsy. Such cases are known as incidental LB disease (ILBD). Depending on the age of the study population, between 3.8 and $30 \%$ of unselected general autopsied brains have been found to have ILBD $[27,32,33]$. This occurrence of ILBD is quite similar to the reported 24\% prevalence of LB in ET cases [18] and thus may be incidental.

In an autopsy study of 24 ET cases and 21 controls, Shill et al. observed ILBD in $12 \%$ of ET patients and $9.5 \%$ of the neurologically normal controls [34]. As in the previous reports [18], in most cases the LB were localized to the lower brainstem [34]. Adler et al. compared the clinical features in the pathologically identified ILBD cases with those who did not have ILBD [33]. The frequency of ET was similar in the two groups $-46 \%$ in the ILBD cases and $40 \%$ in those without ILBD [33]. Thus, ILBD does not appear to be a pathological finding exclusive to ET.

It is generally believed that ILBD is a preclinical pathological state of PD. Therefore, the prevalence of ILBD should parallel the prevalence of PD. If the PD prevalence in the ET cases was 24-times higher than in the general population [30], and we were to use the lowest reported ILBD rate $(3.8 \%)$, more than $91 \%$ of the ET cases should have ILBD at autopsy. Thus, despite the two autopsy studies reporting prevalence of brainstem LB inclusions in 15 [20] and $24 \%$ [18] of the ET brains, those numbers are well within the range of ILBD reported in the general population $[27,32,33,35]$. This leads to the conclusion that the presence of LB in the lower brainstem is not the pathological finding characteristic of ET [27,33-35].

In addition to the evidence that the LB inclusions are not more common in ET than in the general population, there is biochemical evidence 
that separates the ET cases from ILBD cases. Beach et al. observed that striatal tyrosine hydroxylase $(\mathrm{TH})$ levels were reduced in the ILBD brains compared with the normal controls [32]. This observation is consistent with the theory that ILBD is a preclinical pathological state of PD. The same group studied TH levels in the brains of ET cases without ILBD and in normal controls and found no difference in $\mathrm{TH}$ levels between the two groups [36]. These data support a neurochemical difference in the striatal TH levels between ILBD and ET.

Louis postulated that LC neurons synapse with cerebellar PC dendrites and the presence of LB inclusions in the LC leads to PC dysfunction, thereby producing ET symptomatology [2] . LB inclusions in the LC are a feature of Braak stage $2 \mathrm{PD}$ pathology [37]. If LB inclusions in the LC were the pathological basis of tremor production in ET, then one would expect that every PD patient at Braak stage 2 would manifest an ET-like tremor, but the tremor in PD is not evident until Braak stage 3.

Another consideration is the length of time that the LB inclusions may remain localized to the LC (Braak stage 2) without progression to stage 3. It is estimated that such evolution occurs in approximately 10 years [38]. In the cases reported by Louis et al., the LB inclusions were localized to the LC and the lower brainstem after a mean ET duration of 28 years [18]. We believe that the LB inclusions in these cases had a more recent onset and were incidental rather than causally linked to ET.

The cerebellum is the most common site of pathology implicated in the pathogenesis of ET $[17,18,20]$. We will discuss the evidence pertinent to this.

\section{Similarities between ET \& clinical} manifestations of cerebellar pathology

ET is a clinically heterogenous disorder [6,7,39], in which all patients experience an action tremor. There are reports that in some ET cases patients also experience gait difficulty $[6,40,41]$. This combination of action tremor and gait disorder suggests that ET is a possible cerebellar disorder. Action tremor in the ET cases has two attributes: PT, which is seen in a body part held against gravity such as holding the arms at a right angle (perpendicular) to the body and KT, which manifests during movement, such as an attempt to drink from a cup or the finger-to-nose test. When the $\mathrm{KT}$ is accentuated at the end movement, it is known as intention tremor. The PT and KT may be of equal severity or one may be more pronounced than the other in ET cases [7]. The action tremor of cerebellar disease, however, only has the kinetic characteristic [7]. It has been reported that when an ET patient suffers a cerebellar stroke [42] or cerebellar hemorrhage [43], the PT resolved $[42,43]$ and the KT was modified by ataxia and dysmetria [43]. Thus, an indiscriminately destructive cerebellar pathology is not the likely basis of the typical ET manifestations.

There has been a recent focus on the degeneration of cerebellar PC neurons [2,16-18,44-46] and its sequelae [16-18] in the pathogenesis of ET.

\section{PC degeneration \& ET}

Alcohol produces a symptomatic improvement in ET but is toxic to the cerebellar PC $[6,47,48]$. The symptomatic benefit of alcohol on tremor is neither universal nor exclusive to ET. Rajput et al. studied the effect of alcohol on tremors [48]. They observed that alcohol had symptomatic benefit on action tremor in the majority of ET cases but also in nearly half of the PD cases and even in some patients with cerebellar ataxia. An epidemiological study from Spain observed that prior alcohol consumption significantly increased the risk of ET, which was attributed to cerebellar PC toxicity [44]. Another large study from Italy reported that wine consumption significantly reduced the risk of ET [49]. They attributed the benefit to the antioxidant effect of wine. Most of the autopsy reports on ET did not find heavy alcohol use in the ET cases $[4,18]$. This could be attributed to autopsy case selection bias. On balance, the use of alcohol and the risk of developing ET cannot be established conclusively. Contradictory epidemiological observations are a concern to many. Editors of Annals of Neurology note "...epidemiology has become progressively more susceptible to irreproducibility..." [50]. Cerebellar imaging studies have also produced contradictory results [6].

\section{Cerebellar pathology in ET}

Since the topic of this paper is neurodegeneration as the pathological basis of ET, we will focus on the neuropathology literature dealing with PC neuronal loss in this disorder.

Most standard neuropathology studies have reported no consistent abnormalities in the cerebellum of ET patients [6]. Rajput et al. studied 20 ET brains and observed PC loss in two $(10 \%)$ - one had a history of strokes and the 
other had diabetes and coronary artery disease [4]. Shill et al. studied 24 ET and 21 neurologically normal control brains [34]. Seven of the 24 (29\%) ET cases had some cerebellar pathology but none had cerebellar hemisphere atrophy. Three of those cases had Bergmann glia proliferation and four had incidental tauopathy including one with a progressive supranuclear palsy diagnosis. The torpedo formation was not notable in any of their cases [34]. Louis et al. studied ten ET and 12 normal control brains [19]. Six out of the ten ET cases in that study had LB inclusions including two with pathological findings of PD [19]. None of those ten cases had PC loss or gliosis in the cerebellum [19]. Louis and colleagues have subsequently published several detailed studies which included morphometry, showing cerebellar pathology in those ET patients who did not have lower brainstem LB inclusions [17,18,20,45,51]. The PCs were counted by a blinded rater when the nucleus of the neuron was identified. The main findings in those studies were that most ET patients demonstrated a loss of PCs and its sequelae including 'PC torpedoes' [18], Bergmann gliosis and 'hairy baskets' [51]. One of those comprehensive studies included 33 ET and 21 normal controls [18]. It concluded that "all ET brains had either LB or cerebellar pathological changes" [18]. A large majority (76\%) were noted to have cerebellar pathology, characterized by PC loss and its sequelae [18]. In two cases dentate nucleus neuronal loss was detected [18]. Another study by this group performed detailed PC counts in 14 ET brains - eight without LB inclusions, six with brainstem LB inclusions, and 11 normal controls [17]. The mean PC count in the eight cases without LB inclusions were significantly lower compared with the ET cases with LB inclusions and the normal controls [17]; there was no overlap in PC counts between the ET cases without LB and any of the other two groups [17]. Based on those two observations $[17,18]$, Louis classified the ET patients into two groups: 'cerebellar ET' and 'LB variant of ET' [2]. However, those two papers although published during different calendar years $[17,18]$, appear to have been under preparation at the same time, and we assume these were not independent observations but rather that the larger study [18] included the cases reported in the smaller study [17]. In the larger study, the 'cerebellar ET' cases had a mean 31\% reduction in PC counts compared with the controls, however, 17 of those 25 (68\%) cases had PC counts in the normal range [18]. Thus, all the ET patients who did not harbor brainstem LB inclusions did not show PC loss [18]. Combined, these observations are not consistent with PC degeneration being the pathological basis of ET. To extrapolate that PC loss is the pathological basis of ET by using mean PC counts in a group of ET cases where more than two-thirds of ET cases had a normal complement of PCs is seriously flawed [18]. If PC degeneration was the pathological basis of 'cerebellar ET' all such cases would be expected to manifest marked PC loss, however only $32 \%$ of these cases had some PC loss $[18,46]$. Another important consideration is that PC loss is a part of normal aging $[17,18]$. This bias cannot be excluded when the mean PC counts are compared in different groups.

Ideally, we should count all the PCs in the cerebellum to determine if PC loss is the basis of ET manifestations. However, no such technology exists. Therefore, we rely on the sampling of cerebellar tissue to count the PCs.

In an attempt to replicate the observations in previous reports $[17,18,20]$, Rajput et al. performed PC counts in a total of $15 \mathrm{ET}$ and control brains and found no evidence that PC loss was the pathological basis of ET [16]. However, the study was criticized for the small 'total sample size' [45]. Such criticism would be justified if the objective had been to establish a significant correlation between PC loss and ET for an epidemiological study. However, the goal of all these studies was to determine if PC loss is the pathological basis of ET [16-18]. Correlations must not be confused with causation. For every disease the cause must exist (i.e., there is $100 \%$ correlation regardless of the study sample size).

To address that criticism, Rajput et al. enlarged their study to a total of 59 cases including ET patients, PD controls and normal controls [52]. None of the ET cases demonstrated lower brainstem LB inclusions and were thus comparable to the 'cerebellar ET' cases studied by Axelrad et al. [17] and Louis et al. [18]. The ET cases and the PD controls were followed by the same two neurologists (AH Rajput and A Rajput) at the Movement Disorders Clinic Saskatchewan (SK, Canada). The normal controls had no history of neurological disease and had normal brain histology. Unlike previous reports where the PCs were counted only when the neuronal nucleus was visible [17,18], they used three different markers for PC identification [16,52]. Those included: a section through any part of the PC, through the nucleus of the PC and through the nucleolus of 


\begin{tabular}{|c|c|c|c|c|c|}
\hline \multirow[t]{2}{*}{ Diagnosis } & \multicolumn{5}{|c|}{ Mean count (standard deviation) } \\
\hline & $\begin{array}{l}\text { Individuals } \\
\text { (n) }\end{array}$ & $\begin{array}{l}\text { Any part of PC } \\
\text { recognized }\end{array}$ & $\begin{array}{l}\text { PC nucleus } \\
\text { recognized }\end{array}$ & $\begin{array}{l}\text { PC nucleoli } \\
\text { recognized }\end{array}$ & p-value \\
\hline ET & 12 & $64.8(18.3)$ & $31.3(9.7)$ & $20.0(6.3)$ & NS \\
\hline PD controls & 41 & $56.2(12.7)$ & $29.9(8.1)$ & $16.8(6.0)$ & NS \\
\hline $\begin{array}{l}\text { Neurologically normal } \\
\text { controls }\end{array}$ & 6 & $58.0(13.3)$ & $26.2(8.0)$ & $18.3(6.4)$ & NS \\
\hline
\end{tabular}

the PC. All PC counts were carried out on five randomly chosen $100 \times$ power microscopic fields when each field contained a single length of PC through the entire $2 \mathrm{~mm}$ diameter of the microscopic field [52]. Those five PC counts were added to the total number of PCs [52]. The PC counts in all three groups of cases were performed by the Royal College of Physicians and Surgeons of Canada Board certified neuropathologist blinded to the clinical diagnosis [52].

Table 1 shows the mean PC counts in the ET and the two control groups. Although, by each counting method, the PC numbers were

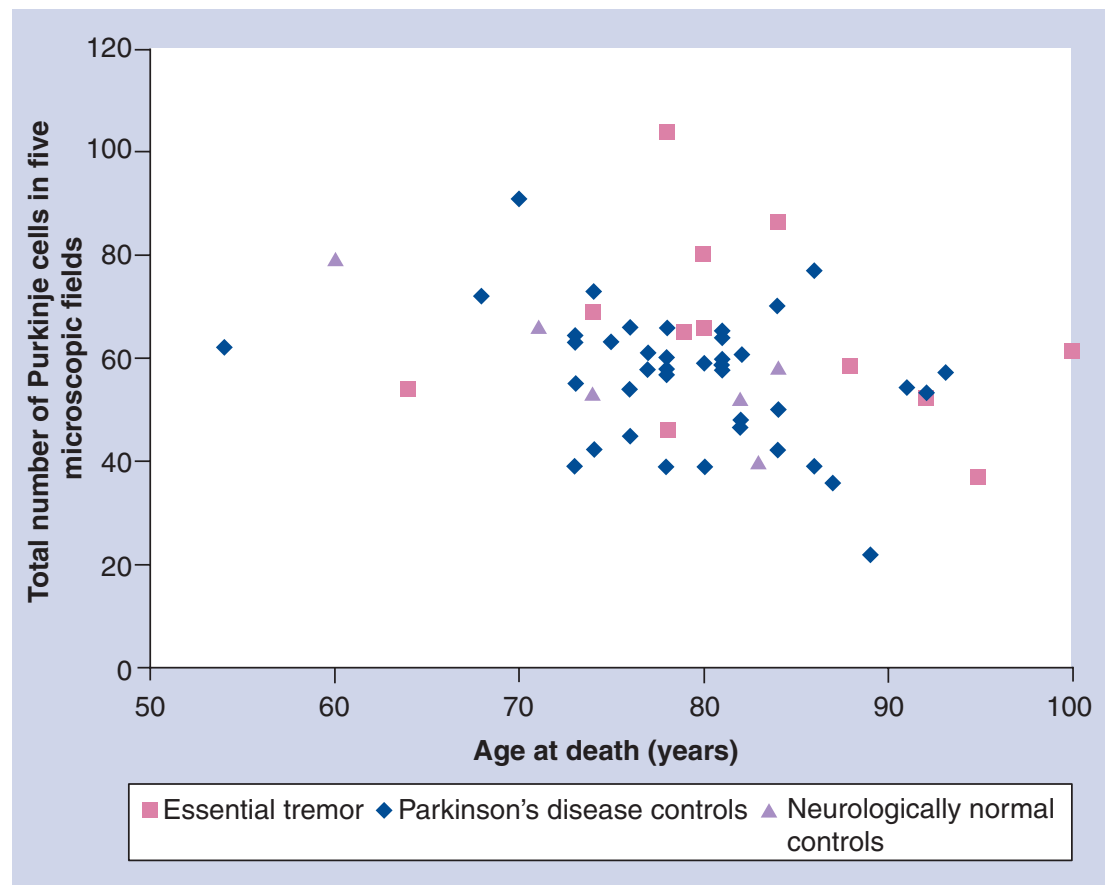

Figure 1. Purkinje cells sectioned through any part of the cell body. Total number of Purkinje cells in five 100x power microscopic fields, when any part of the neuron was identified, and age at death in essential tremor and Parkinson's disease and neurologically normal control brains. There is no difference between the essential tremor and the two control groups of the same age.

Adapted with permission from [52]. marginally higher in the ET group than in the control groups, the difference was not significant. This group also analyzed the PC counts by age of the subjects [52]. Figure 1 shows the PC counts in the three groups when any part of the neuron with or without nucleolus or nucleus was recognized and age at death. Figure 2 shows PC counts based on the identification of the nucleus of the PC and Figure 3 shows PC counts based on nucleolus identification. There was no difference in the PC counts between the ET and control groups of the same age by any of the three counting methods.

Another way to assess neurodegeneration as the basis of ET is to study the critical level of PC loss needed for clinical onset. There is no literature indicating that a threshold PC loss leads to ET manifestations. If PC loss were the pathological basis of ET, the reduced number of PCs would be evident at the time of the first ET manifestation and it would accentuate with time. In the study by Louis et al. [18], 68\% of the 'cerebellar ET' cases had a normal PC count, even after a mean 34-year duration of ET symptoms [46]. Rajput et al. detected no difference in PC counts between ET and the controls after a mean of 34 years of ET symptoms [52]. These data indicate that there is no threshold of PC loss that coincides with ET onset and unlike the neurodegenerative disease $[12,14]$, the PC loss is not accelerated in the ET cases (Figures 1-3). The neuropathology literature can also be analyzed by assessing the clinical consequences of PC loss. The burden of pathology should be reflected in the severity of clinical manifestations $[12,14]$. If PC loss accounted for ET manifestations, individuals with more pronounced loss would probably have the most severe clinical features. Rajput et al. found no association between the degree of PC loss and the severity of tremor or the number of anatomical sites involved in ET [16]. Isolated case studies indicate that even when there is 
complete loss of cerebellar PC the patients do not show features of ET [53,54]. Ishida et al. reported one case with glutamic acid antibody that had complete cerebellar PC loss and Bergmann gliosis [53]. This patient had marked cerebellar ataxia but no features of ET during a 5-year followup. Verschuuren et al. reported a patient (case 1) with ovarian adenocarcinoma who developed progressive limb and truncal ataxia but had no ET manifestations [54]. At autopsy there was a complete loss of PCs. These two cases illustrate that even the extreme PC depletion does not lead to clinical features of ET.

The clinicopathological literature to date does not support the conclusions made by Louis and colleagues; that PC degeneration is the pathological basis of ET $[2,17,18]$. In their recent communication, they note that "While cerebellar PC loss is certainly not pathognomonic of ET ... warrants further investigation" [45]. We agree with that conclusion. At this time we need to consider all possibilities in the pathogenesis of ET.

We have considered multiple sources of clinicopathological evidence to determine neurodegeneration in ET. The literature clearly indicates that neither the brainstem LB inclusions nor cerebellar PC degeneration is the pathological basis of ET. Therefore, the use of the terms 'LB variant of ET' and 'cerebellar ET' should be discontinued.

\section{Considering alternatives to neurodegeneration in ET pathophysiology}

It has been postulated that ET may be consequent to metabolic dysfunction, which triggers abnormal oscillations in the cerebello-thalamocortical motor circuit [6]. Some other progressively disabling neurological disorders are known for not having neurodegeneration (e.g., untreated dopa-responsive dystonia) which, like PD, improves with levodopa but unlike PD has no substantia nigra neuronal loss [55,56].

GABA is the most common inhibitory substance in the brain and several GABAergic drugs are known to improve ET $[2,47,57]$. Altered GABA function has been considered the possible basis of the motor circuit dysfunction in ET and brain GABA function in ET has been investigated by several groups [58-60].

Boecker et al., who studied GABA , receptors in ET and control subjects using PET, detected a decline in GABA function in the dentate

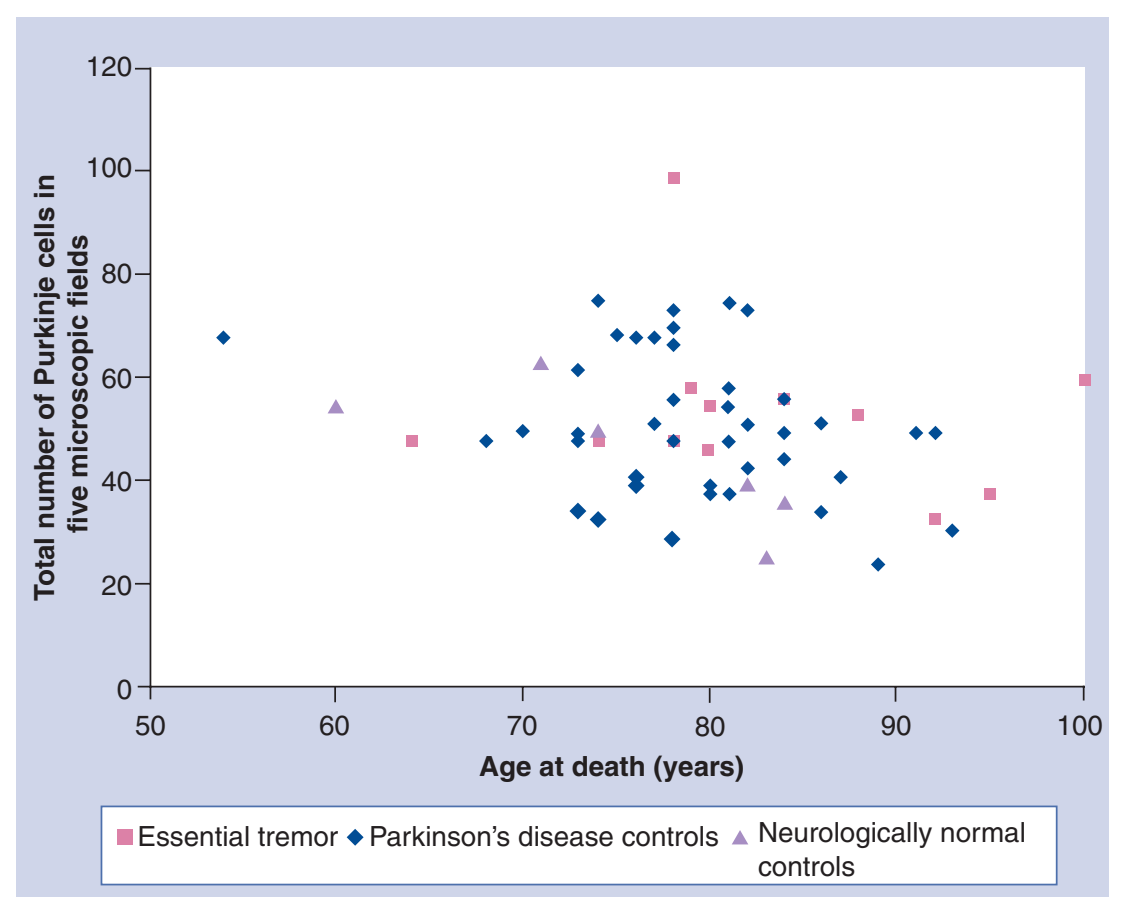

Figure 2. Purkinje cells sectioned through the nucleus. Total number of Purkinje cells from five $100 \times$ power microscopic fields, when the nucleus of the neuron was identified, and age at death in essential tremor and Parkinson's disease and neurologically normal control brains. There is no difference between essential tremor and the two control groups of the same age.

Adapted with permission from [52].

nucleus, ventrolateral thalamus and the lateral premotor cortex [58]. Paris-Robidas et al. studied cerebellar GABA receptors in ten ET, ten PD and 16 normal control brains [59]. They observed a $35 \% \mathrm{GABA}_{\mathrm{A}}$ receptor reduction in the dentate nucleus of ET patients, compared with the normal and the PD controls. They also detected a $22 \%$ decrease of $\mathrm{GABA}_{\mathrm{B}}$ receptors in the ET dentate nucleus of ET cases compared with that in the dentate nucleus of normal controls and a $31 \%$ decline compared with PD controls dentate nucleus [59]. However, they found no change in the $\mathrm{GABA}_{\mathrm{A}}$ or $\mathrm{GABA}_{\mathrm{B}}$ receptors in the cerebellar cortex [59]. $\mathrm{GABA}_{\mathrm{B}}$ concentration had an inverse relationship with the duration of ET symptoms, suggesting that this abnormality was progressive and was of a long duration [59]. More recently, Shill et al. reported reduced parvalbumin (GABA interneuron marker) in the $\mathrm{LC}$ region of the pons in ET brains [60]. Importantly, however, this reduction was mostly accounted for by the older age of onset of ET, whereas those with 'classic' ET had normal parvalbumin levels, suggesting that typical ET is not a neurodegenerative disease involving the GABAergic neurons. 


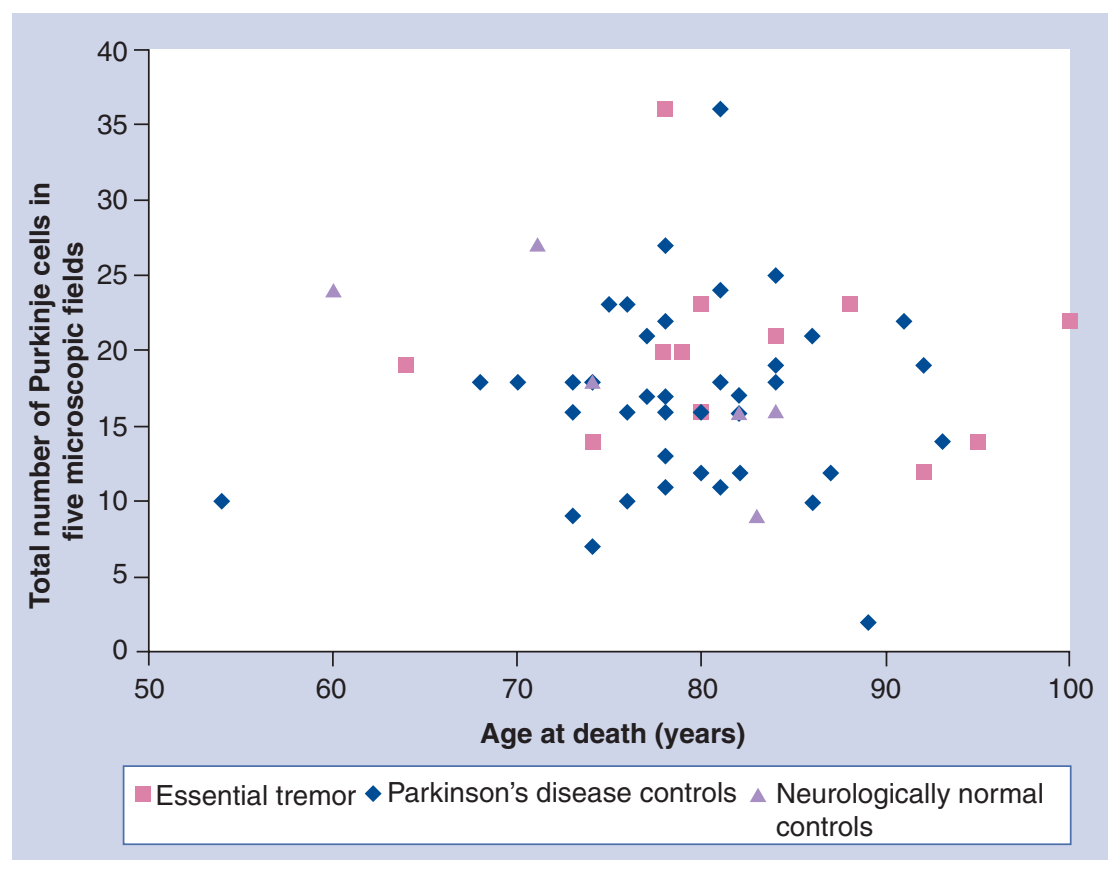

Figure 3. Purkinje cells sectioned through the nucleolus. Total number of Purkinje cells from five 100x power microscopic fields, when part of the nucleolus of the neuron was identified, and age at death in essential tremor brains and Parkinson's disease and neurologically normal control brains. There is no difference between essential tremor and the two control groups of the same age.

Adapted with permission from [52]. need to be considered when trying to gain a better understanding of the pathophysiology of ET. Finally, it may well be the case that multiple mechanisms are involved in causing ET.

\section{Future perspective}

Given the current level of scientific interest in ET, the next 10 years will see significant advances in this field.

Neither of the two proposed types of neurodegeneration form the pathological basis of ET. Evidence is now emerging that there are brainbased biochemical abnormalities that result in altered functions and manifest as a tremor. Not all the ET cases will fit one biochemical profile; we expect several different abnormalities will be identified.

We predict that there will be future studies focused on large ET kindreds to identify a genetic basis of the disorder. We anticipate that several different genetic abnormalities will be identified and there will be imaging studies aimed at abnormalities in brain neurotransmitters, including GABA. The above two types of studies will be most informative if they are performed in the same cohort. Such patients will then be followed and eventually autopsy studies will be performed for biochemical abnormalities and to determine if there is a localized gene expression that accounts for the clinical manifestations. The patients will be classified based on genotyping and different clinical profiles will be established in those genotypes. Such studies of large families will then be replicated in sporadic ET cases. Based on those studies, there will be targeted drugs devised for different subtypes of ET.

\section{Acknowledegments}

The authors are grateful to $L$ Beatty for the production of this manuscript and M Rajput for the figures and table.

in the thalamus or the lateral premotor corte to support the abnormal GABA function found with PET scanning [58].

\section{Conclusion}

After careful review of the published literature on clinical, neuropathology and neurochemical findings in ET, we find no evidence to support the notion that ET is a neurodegenerative disorder - either characterized by the lower brainstem LB inclusions or by cerebellar PC degeneration. Further studies are needed with larger numbers of ET cases (hereditary, sporadic and senile) and agematched controls using standardized methods of performing the evaluations [6]. All the alternatives

\section{Financial \& competing interests disclosure}

Financial support was received from the Regina Curling Classic for Parkinson Research, the Greystone GolfClassic for Parkinson's, the Royal University Hospital Foundation, the Michael J Fox Foundation for Parkinson's Research, the Arizona Biomedical Research Commission and the National Institute of Neurological Disorders and Stroke. The authors have no other relevant affiliations or financial involvement with any organization or entity with a financial interest in or financial conflict with the subject matter or materials discussed in the manuscript apart from those disclosed.

No writing assistance was utilized in the production of this manuscript. 


\section{References}

Papers of special note have been highlighted as:

- of interest

- of considerable interest

1 Louis ED, Ford B, Lee H, Andrews H, Cameron G. Diagnostic criteria for essential tremor - a population perspective. Arch. Neurol. 55, 823-828 (1998).

2 Louis ED. Essential tremors: a family of neurodegenerative disorders? Arch. Neurol. 66(10), 1202-1208 (2009).

- Makes the argument that essential tremor (ET) is a neurodegenerative disease.

3 Dogu O, Sevim S, Camdeviren $\mathrm{H}$ et al. Prevalence of essential tremor door-to-door neurologic exams in Mersin Province, Turkey. Neurology 61, 1804-1806 (2003).

4 Rajput A, Robinson C, Rajput AH. Essential tremor course and disability: a clinicopathological study of 20 cases. Neurology 62, 932-936 (2004).

-. Long-term study of ET patients followed by the same two neurologists and autopsy verification - provides extensive information on ET for clinicians.

5 Fekete R, Jankovic J. Revisiting the relationship between essential tremor and Parkinson's disease. Mov. Disord. 26, 391-398 (2011).

6 Deuschl G, Elble R. Essential tremor - neurodegenerative or nondegenerative disease towards a working definition of ET. Mov. Disord. 24(14), 2033-2041 (2009). Good review by two leading experts.

7 Elble RJ. Diagnostic criteria for essential tremor and differential diagnosis. Neurology 54(11 Suppl. 4), S2-S6 (2000).

8 Rajput AH, Offord KP, Beard CM, Kurland LT. Essential tremor in Rochester, Minnesota: a 45-year study. J. Neurol. Neurosurg. Psychiatr. 47, 466-470 (1984).

9 Larsen TA, Calne DB. Essential tremor. Clin. Neuropharmacol. 6(3), 185-206 (1983).

10 Rajput AH. Essential tremor that is not 'benign'. Trans. Can. Congress Neurol. 55, 151 (1976).

11 Louis ED, Okun MS. It is time to remove the 'benign' from the essential tremor label. Parkinsonism Relat. Disord. 17, 516-520 (2011).

12 Lees AJ. The Parkinson chimera. Neurology 72(Suppl. 2), S2-S11 (2009).

13 Rajput AH, Rozdilsky B, Hornykiewicz O, Shannak K, Lee T, Seeman P. Reversible drug-induced Parkinsonism: clinicopathologic study of two cases. Arch. Neurol. 39, 644-646 (1982).
14 Alvord EC Jr, Forno LS, Kusske JA, Kauffman RJ, Rhodes JS, Goetowski CR. The pathology of Parkinsonism: a comparison of degenerations in cerebral cortex and brainstem. In: Advances in Neurology (Volume 5).

McDowell FH, Barbeau A (Eds). Raven Press, NY, USA, 175-193 (1974).

15 Gelb DJ, Oliver E, Gilman S. Diagnostic criteria for Parkinson disease. Arch. Neurol. 56, 33-39 (1999).

16 Rajput AH, Robinson CA, Rajput ML, Rajput A. Cerebellar Purkinje cell loss is not pathognomonic of essential tremor. Parknisonism Relat. Disord. 17, 16-21 (2011).

17 Axelrad JE, Louis ED, Honig LS et al. Reduced Purkinje cell number in essential tremor. Arch Neurol. 65(1), 101-107 (2008).

- Cerebellar Purkinje cell (PC) counts in a small number of ET cases and in controls - showing PC loss is restricted in 'cerebellar ET'.

18 Louis ED, Faust PL, Vonsattel JP et al. Neuropathological changes in essential tremor: 33 cases compared with 21 controls Brain 130, 3297-3307 (2007).

- Large detailed pathology study of ET cases from multiple centers demonstrating that mean PC levels in 'cerebellar ET' cases were reduced compared with controls.

19 Louis ED, Vonsattel JPG, Honig LS, Ross GW, Lyons KE, Pahwa R. Neuropathologic findings in essential tremor. Neurology 66, 1756-1759 (2006).

20 Louis ED, Faust PL, Vonsattel JPG et al. Torpedoes in Parkinson's disease, Alzheimer's disease, essential tremor, and control brains. Mov. Disord. 24(11), 1600-1605 (2009).

21 Duvoisin RC, Golbe LI. Toward a definition of Parkinson's disease. Neurology 39, 746 (1989).

22 Rajput AH, Rozdilsky B, Rajput A. Accuracy of clinical diagnosis in Parkinsonism - a prospective study. Can. J. Neurol. Sci. 18, 275-278 (1991)

- First publication in the literature showing that clinical diagnosis of Parkinson's disease (PD) at initial evaluation is incorrect in 35\% of cases and at final assessment is inaccurate in $24 \%$ of cases. Study was based on cases followed longitudinally by the same neurologist. Similar results were later reported by other centers.

23 Jellinger $\mathrm{K}$. The pathology of Parkinsonism. In: Movement Disorders 2. Marsden CD, Fahn $S$ (Eds). Butterworths and Co., London, UK, $124-165$ (1987)
24 Marshall V, Grosset DG. Role of dopamine transporter imaging in the diagnosis of atypical tremor disorders. Mov. Disord. 18(Suppl. 7), S22-S27 (2003).

25 Isaias IU, Marotta G, Hirano S et al. Imaging essential tremor. Mov. Disord. 25(6), 679-686 (2010).

26 Stoessl AJ. Neuroimaging in Parkinson's disease. Neurotherapeutics 8, 72-81 (2011).

27 Adler CH, Shill HA, Beach TG. Essential tremor and Parkinson's disease: lack of a link. Mov. Disord. 26, 372-377 (2011).

- Good review showing that ET and PD are different disorders.

28 Moghal S, Rajput AH, D'Arcy C, Rajput R. Prevalence of movement disorders in elderly community residents. Neuroepidemiology 13 , 175-178 (1994)

29 Cleeves L, Findley LJ, Koller W. Lack of association between essential tremor and Parkinson's disease. Ann. Neurol. 24, 23-26 (1988).

30 Geraghty JJ, Jankovic J, Zetusky WJ. Association between essential tremor and Parkinson's disease. Ann. Neurol. 17, 329-333 (1985).

31 Rajput AH, Rozdilsky B, Ang L, Rajput A. Significance of Parkinsonian manifestations in essential tremor. Can. J. Neurol. Sci. 20, 114-117 (1993).

32 Beach TG, Adler CH, Sue LI et al. Reduced striatal tyrosine hydroxylase in incidental lewy body disease. Acta Neuropathol. 115, 445-451 (2008).

33 Adler $\mathrm{CH}$, Connor DJ, Hentz JG et al. Incidental lewy body disease: clinical comparison to a control cohort. Mov. Disord. 25(5), 642-646 (2010).

34 Shill HA, Adler CH, Sabbagh MN et al. Pathologic findings in prospectively ascertained essential tremor subjects. Neurology 70, 1452-1455 (2008)

35 Quinn NP, Schneider SA, Schwingenschuh P, Bhatia KP. Tremor - some controversial aspects. Mov. Disord. 26, 18-23 (2011).

36 Shill HA, Adler CH, Beach TG, Sue LI, Campbell NA, Walker DG. Striatal tyrosine hydroxylase in autopsied patients with essential tremor. Neurology 72(Suppl. 3), A100 (2009).

37 Braak H, Del Tredici K, Rub U, de Vos RA, Jansen Steur EN, Braak E. Staging of brain pathology related to sporadic Parkinson's disease. Neurobiol. Aging 24(2), 197-211 (2003).

38 Hawkes $\mathrm{CH}$, Del Tredici K, Braak H. A timeline for Parkinson's disease. Parkinsonism Relat. Disord. 16, 79-84 (2010). 
39 Jankovic J. Essential tremor: clinical characteristics. Neurology 54(Suppl. 4), S21-S25 (2000).

40 Singer C, Sanchez-Ramos J, Weiner WJ. Gait abnormality in essential tremor. Mov. Disord. 9(2), 193-196 (1994).

41 Deuschl G, Wenzelburger R, Loffler K, Raethjen J, Stolze H. Essential tremor and cerebellar dysfunction - clinical and kinematic analysis of intention tremor. Brain 123, 1568-1580 (2000).

42 Dupuis MJM, Delwaide PJ, Boucquey D, Gonsette RE. Homolateral disappearance of essential tremor after cerebellar stroke. Mov. Disord. 4(2), 183-187 (1989).

43 Rajput AH, Maxood K, Rajput A. Classic essential tremor changes following cerebellar hemorrhage. Neurology 71, 1739-1740 (2008).

44 Louis ED, Benito-Leon J, Bermejo-Pareja F. Population-based study of baseline ethanol consumption and risk of incident essential tremor. J. Neurol. Neurosurg. Psychiatr. 80, 494-497 (2009).

45 Louis ED, Faust PL, Vonsattel JP. Purkinje cell loss is a characteristic of essential tremor. Parkinsonism Relat. Disord. 17, 406-409 (2011).

46 Rajput AH, Rajput A. Significance of cerebellar Purkinje cell loss to pathogenesis of essential tremor. Parkinsonism Relat. Disord. 17, 410-412 (2011).

47 Koller WC, Hristova A, Brin M. Pharmacologic treatment of essential tremor. Neurology 54(Suppl. 4), S30-S38 (2000).

48 Rajput AH, Jamieson H, Hirsch S, Quraishi A. Relative efficacy of alcohol and propranolol in action tremor. Can. J. Neurol. Sci. 2, 31-35 (1975).

49 Nicoletti A, Mostile G, Cappellani R et al. Wine drinking and essential tremor: a possible protective role. Mov. Disord. 26, 1310-1315 (2011).

50 Johnston SC, Hauser SL. Diagnosis and prognosis unclear for scientific manuscripts. Ann. Neurol. 69, A9-A10 (2011).

51 Erickson-Davis CR, Faust PL, Vonsattel JP, Gupta S, Honig LS, Louis ED. 'Hairy Baskets' associated with degenerative Purkinje cell changes in essential tremor.

J. Neuropathol. Exp. Neurol. 69(3), 262-271 (2010).

52 Rajput AH, Robinson CA, Rajput ML, Robinson SL, Rajput A. Essential tremor is not dependent upon cerebellar Purkinje cell loss. Parkinsonism Relat. Disord. doi:10.1016/j. parkreldis.2012.01.013 (2012) (Epub ahead of print).

-1 Large autopsy study on ET and controls followed at one institution by the same two movement disorder neurologists, detailing cerebellar PC counts by a neuropathologist; demonstrated that PC loss is not the pathological basis of ET.

53 Ishida K, Mitoma H, Wada Y et al. Selective loss of Purkinje cells in a patient with anti-glutamic acid decarboxylase antibodyassociated cerebellar ataxia. J. Neurol. Neurosurg. Psychiatr. 78, 190-192 (2007).

54 Verschuuren J, Chuang L, Rosenblum MK et al. Inflammatory infiltrates and complete absence of Purkinje cells in anti-Yo-associated paraneoplastic cerebellar degeneration. Acta Neuropathol. 91, 519-525 (1996).
55 Rajput AH. Levodopa in dystonia musculorum deformans. Lancet 1, 432 (1973).

56 Rajput AH, Gibb WRG, Zhong XH et al. Dopa-responsive dystonia: pathological and biochemical observations in a case. Ann. Neurol. 35, 396-402 (1994).

57 Zesiewicz TA, Elble RJ, Louis ED et al. Evidence-based guideline update: treatment of essential tremor. Report of the Quality Standards Subcommittee of the American Academy of Neurology. Neurology 77, 1752-1755 (2011).

58 Boecker H, Weindl A, Brooks DJ et al. GABAergic dysfunction in essential tremor: an ${ }^{11} \mathrm{C}$-flumazenil PET study. J. Nucl. Med. 51(7), 1030-1035 (2010).

-. Very good study of PET scanning in ET, showing GABA dysfunction in ET at multiple sites including cerebellar dentate nucleus.

59 Paris-Robidas S, Brochu E, Sintes M et al. Defective dentate nucleus GABA receptors in essential tremor. Brain 135, 105-116 (2012).

-. Detailed study of ET and control brains showing decline in GABA receptors in the dentate nucleus in ET compared with PD and normal controls, but no GABA changes in the cerebellar cortex.

60 Shill HA, Adler CH, Beach TG et al. Brain biochemistry in autopsied patients with essential tremor. Mov. Disord. 27(1), 113-117 (2012).

- Detailed study showing reduced parvalbumin (GABA interneuron marker) in the locus ceruleus region of ET compared with controls. 